\title{
88. Contraction of Cultured Chick Myotube before Acquisition of Cell Membrane Excitability
}

\author{
By Koji SaIto and Eijiro Ozawa \\ Division of Cell Biology, National Center for Nervous, Mental \\ and Muscular Disorders, Kodaira, Tokyo 187 \\ (Communicated by Setsuro EbaSHI, M. J. A., Dec. 12, 1983)
}

Excitation-contraction coupling has been studied in skeletal muscle of adult vertebrates (Ebashi and Endo, 1968). Little is known of how the coupling develops during myogenesis, although changes in excitability of muscle cell membrane have been studied in developing chick, chick embryos and cultured chick cells (Fischbach et al., 1971; Kano and Shimada, 1973; Kano, 1975). The development of contractility, however, has not been investigated in myogenic cells. As a first step in investigating the development of excitation-contraction coupling, we developed a method to record the contraction of cultured chick myotubes. In this communication, we describe the method and the result that contractility differentiated before the differentiation of excitability in cell membrane of cultured chick myotubes.

Methods. Myotubes were prepared from 10-12 day old chick embryos as described previously (Kimura et al., 1981). The cells were plated on $35-\mathrm{mm}$ gelatin coated dishes $\left(2 \times 10^{5}\right.$ cells $/$ dish $)$ and incubated in a humidified atmosphere containing $5 \% \mathrm{CO}_{2}$ at $37^{\circ} \mathrm{C}$. Culture medium was composed of $85 \%$ Eagles' MEM (Nissui Co., Tokyo) and $15 \%$ horse serum (Gibco Lab., Grand Island) containing $20 \mu \mathrm{g} / \mathrm{ml}$ of Fe-bound chick transferrin (conalbumine Type II, Sigma Chemical Co., St. Louis). Chick transferrin was used in place of embryo extract (Ii et al., 1982) because of its less stimulatory effect on fibroblast growth.

Prior to and during the experiments, each culture dish was perfused with a medium of the following composition (mM) ; NaCl, $150 ; \mathrm{KCl}, 2.68 ; \mathrm{CaCl}_{2}, 1.8 ; \mathrm{MgCl}_{2}, 1.05 ;$ glucose, $5.55 ; \mathrm{HEPES}, 2$ $(\mathrm{pH} 7.4)$ and $1 \%$ bovine serum albumin (Fraction V, Sigma Chemical Co., St. Louis). Temperature of the medium in the dish was kept at $36^{\circ} \mathrm{C}$.

A pair of conventional microelectrodes were used for passing current and recording membrane potential changes. The electrodes were filled with $3 \mathrm{M} \mathrm{KCl}$ or $2 \mathrm{M} \mathrm{K}$-acetate which had a resistance of 15-40 M $\Omega$. They were inserted into the myotube on both sides 
of the glass grain in order to be arranged longitudinally to the myotube (see below). Distance between the electrodes was usually less than $50 \mu \mathrm{m}$.

Movement of the myotube was recorded photometrically as shown in Fig. 1. A light guide $(l g)$ with a lamp was inserted into

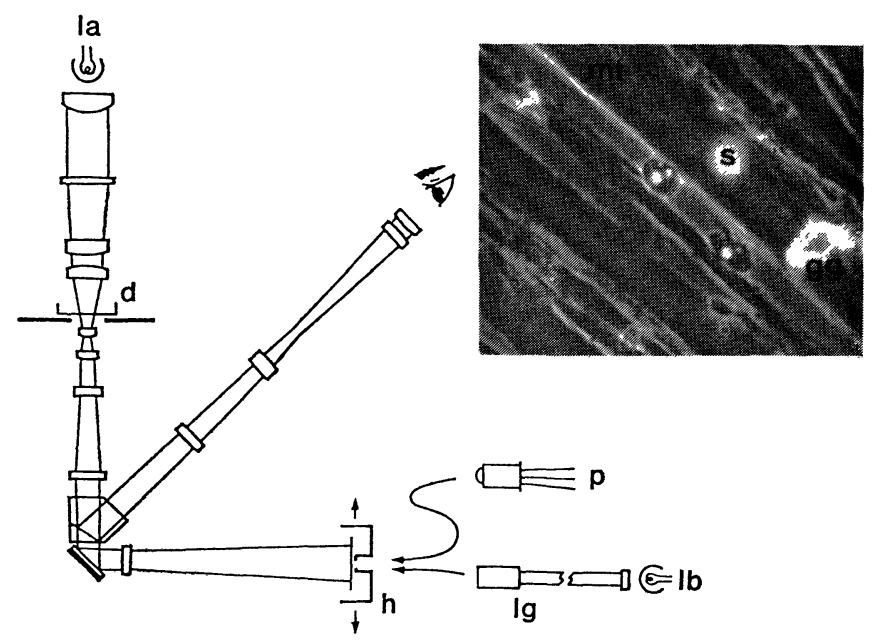

Fig. 1. Illustration of a device to record mechanical activity of a myotube. The photograph shows a bright spot and a glass grain on the myotube to be examined. In the experiment, the bright spot was apposed close to the glass grain. $d$, culture dish; $h$, cylindrical holder; $p$, phototransistor; $l a$ and $l b$, light source; $l g$, light guide; $m t$, myotube; $g g$, glass grain; $s$, bright spot $(18 \mu \mathrm{m}$ in diameter $)$.

a cylindrical holder $(h)$. A culture dish $(d)$ was placed on the turntable of a phase contrast microscope. Glass grains ( $g g$ ) $10-40 \mu \mathrm{m}$ in diameter were scattered on the myotube before the experiment. When the microscope lamp (la) was lit, cells were visualized, and with the lighting of the external lamp (lb), a bright spot (s) $18 \mu \mathrm{m}$ in diameter appeared in the field. By moving the holder, the bright spot was placed at the desired position on the glass grain located on the myotube to be examined (Fig. 1, photograph). The light guide was replaced by a phototransistor $(p)$ which was positioned on the plane where the glass grain was imaged. Movement of the glass grain, caused by contraction of the myotube, was followed by the phototransistor. Current changes generated by the phototransistor were transduced to voltage changes which were displayed on a storage oscilloscope together with membrane potential changes.

Results and discussion. Well developed myotubes showed various types of electrical responses depending on the strength of the 
depolarizing current. Fig. 2a represents responses recorded from a 9-day cultured cell. With small currents, only passive electrical response was elicited. Late depolarization appeared with an increase in current intensity, which was reported to result mainly from an increase in $\mathrm{Ca}^{2+}$ (Kano and Shimada, 1973; Kano, 1975) or $\mathrm{Cl}^{-}$ (Fukuda, 1974; Fukuda et al., 1976) conductance. Abortive and clear spike discharges were obtained with larger current. The spike component is reported to be due to inward current carried by sodium ions. Long duration and small amplitude indicate that the spike discharge is still immature.

Contraction of the myotube was elicited by a passive electrical response when the amplitude of the depolarization exceeded a certain

a
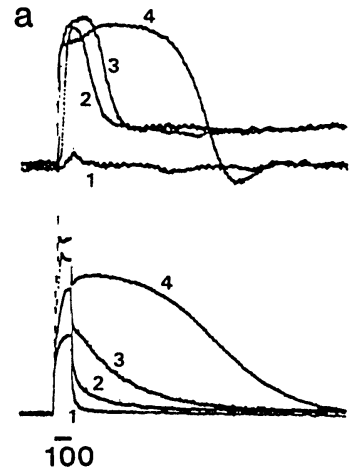

b
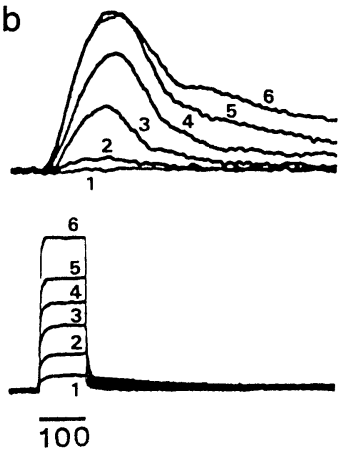

$a^{\prime}$
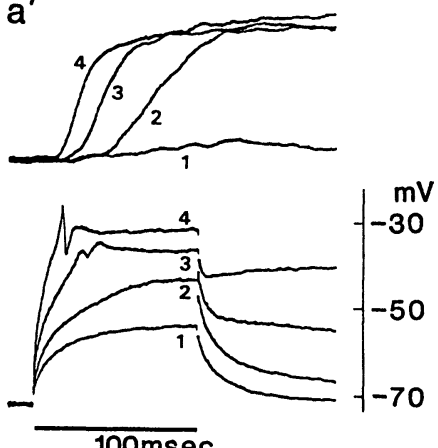

$100 \mathrm{msec}$

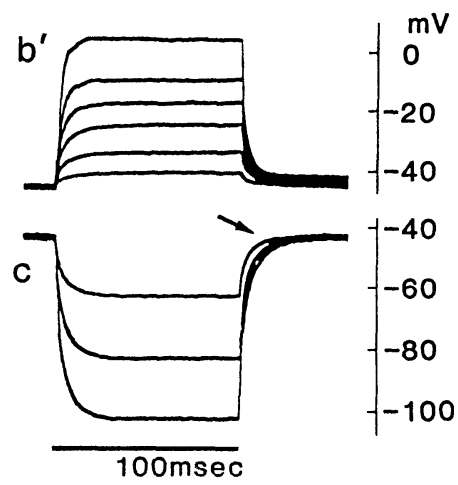

Fig. 2. Electrical and mechanical response of myotubes cultured for $9(a)$ and $4(b)$ days. In $a$ and $b$, upper traces are contractile responses and lower traces are electrical responses to outward current pulses with a duration as shown by the bar. A pair of responses of the same number in both traces were elicited by the same stimulus. Responses $1-4$ in $a$ were elicited by 5 , 7,10 and $15 \mathrm{nA}$ in increasing order, respectively, and 1-6 in $b$ were 2, 5, $10,15,20$ and $30 \mathrm{nA}$. Traces in $a^{\prime}$ and $b^{\prime}$ are fast sweep records of the response in $a$ and in lower traces in $b$, respectively. Traces in $c$ are electrical responses of the 4-day cultured myotube to inward current pulses (10, 20 and $30 \mathrm{nA}$ ). No active response occurs at the arrow. 
threshold (Fig. 2a, upper traces). Latency and rising phase of contraction became short as the rate of rise of electrical response increased with larger current. Time between the half maximum contraction was prolonged by the elongated late depolarization.

Some myotubes were undifferentiated electrophysiologically and did not show the spike discharge and late depolarization when stimulated by large current. These myotubes are more frequently found in young chick embryos than in old ones (Kano, 1975). An example from a 4-day cultured cell is shown in Fig. 2b, which exhibits only passive depolarization following large outward current pulses (lower traces). As this myotube showed low resting membrane potential, a hyperpolarizing current was passed through the membrane to remove any possible inactivation of the late depolarization and spike generation mechanisms (Fischbach et al., 1971). After the end of current pulse, off-response (depolarization or spike discharge; Fukuda et al., 1976) did not appear (Fig. 2c, arrow).

Contraction, however, was elicited in the electrically quiescent myotubes (Fig. 2b, upper traces). When the passive electrical response exceeded a threshold depolarization, contraction appeared in this 4-day cultured cell. Contraction increased in amplitude and duration as the depolarization increased.

These results indicate that contractile mechanisms become functional before the excitability of cell membrane differentiates in cultured chick myotubes.

Acknowledgments. This work was partly supported by grants from the National Center for Nervous, Mental and Muscular Disorders (NCMMD, no. 83-01) of the Ministry of Health and Welfare, and of the Ministry of Education, Science and Culture, Japan.

\section{References}

Ebashi, S., and Endo, M. (1968) : Progr. Biophys. Molec. Biol., 18, 123.

Fischbach, G. D., Nameroff, M., and Nelson, P. G. (1971) : J. Cell. Physiol., 78, 289.

Fukuda, J. (1974) : Science, 185, 76.

Fukuda, J. et al. (1976) : Develop. Biol., 49, 395.

Ii, I., Kimura, I., and Ozawa, E. (1982) : ibid., 94, 366.

Kano, M. (1975) : J. Cell. Physiol., 86, 503.

Kano, M., and Shimada, Y. (1973): ibid., 81, 85.

Kimura, I. et al. (1981) : Proc. Japan Acad., 57B, 200. 